\title{
A new perspective on acoustic rhinometry in terms of standardisation, including the nasal allergen provocation test
}

\author{
Edyta Krzych-Fałta ${ }^{1}$, Olga Kaczyńska², Bolesław Samoliński ${ }^{3}$, Adam Sybilski $^{4}$
}

${ }^{1}$ Nursing Basics Facility, Medical University of Warsaw, Warsaw, Poland

${ }^{2}$ student of the Medical University of Warsaw, Warsaw, Poland

${ }^{3}$ Department of Environmental Risk Prevention, Allergy and Immunology, Medical University of Warsaw, Warsaw, Poland ${ }^{4} 2^{\text {nd }}$ Clinic of Paediatrics, Centre of Postgraduate Medical Education, Warsaw, Poland

Adv Dermatol Allergol 2022; XXXIX (5): 852-855

DOI: https://doi.org/10.5114/ada.2021.109689

\begin{abstract}
Acoustic rhinometry is an objective nasal patency assessment technique. Its cognitive value means that it plays a major role in the nasal allergen provocation test. The optimisation of the measured values and the interpretation of the results of the test-based values are critical. When the assessment is based on the minimal cross-sectional areas, the volumes for the different segments of the rhinometric curve indicate changes in nasal patency in the nasal allergen provocation test. It seems that the points on the rhinometric curve which correspond to the different waves for the nasal cavity accurately reflect the level of response of the mucous membrane of the nasal cavity to an allergen.
\end{abstract}

Key words: acoustic rhinometry, cross sectional areas of the nasal cavity.

\section{Introduction}

Acoustic rhinometry (AR) is a diagnostic method for non-invasive measurement of intranasal spaces. It uses the reflection of a sound wave converted into electrical impulses and generated during the nasal cavity assessment. An AR set consists of a sound generator, a sound tube, a wave-sending microphone, an amplifier, a computer (equipped with analogue-to-digital converters) and an anatomical or conical nasal adapter. The spatial resolution of an AR set falls within the $0.33-0.47 \mathrm{~cm}$ range, and the measurement points mark the rhinometric curve at 3.3-4.7 $\mathrm{mm}$ intervals. Factors such as noise (up to $74 \mathrm{~dB}$ ), temperature fluctuations or lack of tightness of the adapter with a rhinometric tube substantially affect the final result of the test. It is recommended by the Committee on Acoustic Rhinometry Standards of the European Rhinologic Society that AR tests should be performed according to the basic principles of accuracy (accuracy of measurement), repeatability (consistency of measurements), reproducibility (consistency of measurements when changes are made, such as changes of apparatus or the tester), spatial resolution (the capacity to distinguish between spaces along the distance axis) [1].
The general availability of AR, the ease of its application (in children at the age of 5 or older), and the high specificity and sensitivity of the method make it a good tool for assessing the extent of nasal obstruction [2]. It is particularly useful in assessing the bone structure of the nasal cavity (nasal septum deviation) and nasal cavity polyps, in obstructive sleep apnoea diagnosis, as well as in assessing the effectiveness of therapeutic treatment or surgical treatment, and in nasal provocation tests. The last of the applications above, which is particularly recommended in EAACI Position paper on the standardization of nasal allergen challenges [3], adds to the role of this objective nasal patency assessment technique.

\section{The rhinometric curve: interpretation in the broad sense of the term}

The rhinometric curve provides plenty of valuable information that can be used to verify the extent of nasal obstruction or nasal patency on the one hand and is very useful in differential diagnosis of rhinitis conditions on the other. A secondary effect of the reflection of a sound wave is a diagram showing cross-sectional areas at a certain

Address for correspondence: Edyta Krzych-Fałta, Nursing Basics Facility, Medical University of Warsaw, 27 Erazma Ciołka St, 01-445 Warsaw, Poland, phone: +48 2283609 71, e-mail: edyta.krzych-falta@wum.edu.pl Received: 30.06 .2021 , accepted: 8.08.2021. 
depth of the nasal cavity: the $X$ axis shows the length of the nasal cavity and records longitudinal parameters of the nasal cavity, while the $Y$ axis shows the size of the cross-sectional areas at various points of the nasal cavity and records transverse parameters of the cavity (Figure 1). The different points on the AR curve indicate the following: 0 : the start of the nasal cavity, I: the nasal isthmus (isthmus nasi): the peak point of the first AR curve wave, which corresponds to the first narrowing of the nasal cavity, C: the head of the inferior nasal concha (concha nasalis): the peak point of the narrowing of the second AR curve wave, which corresponds to the second narrowing of the nasal cavity, E: the distal section of the head of the inferior nasal concha, F: the top point of the elevation of the $A R$ curve between points I and C. The areas at the above points indicate the following: 0-I: the vestibule of the nose, I-C the distance between the nasal isthmus and the head of the inferior nasal concha, C-E: the end of the nasal concha, $\mathrm{E}-\mathrm{G}$ : the nasopharynx space. Nasal patency is indicated by narrowing of points. The position of these points varies depending on the internal structure of the nasal cavity and/ or the severity of the swelling of the mucous membrane in the nasal cavity. Nasal obstruction in AR is assessed by measuring the $v$ value, namely the minimal cross-sectional area, which is usually the value at point I before the nasal allergen provocation test or at point $C$ after the test; the vv value - by measuring the volume of the nasal cavity; and the vvv value - by measuring the cross-sectional areas (CSAs) of the nasal cavity on the AR curve for different CSA ranges [4].

In the literature, the most frequently assessed area of the nasal cavity is the minimal cross-sectional area (MCA). MCA is represented by a value on the $Y$ axis which corresponds to point I, which is a nasal valve (MCA1: a value of less than $0.28 \mathrm{~cm}^{2}$ indicates nasal septum deviation) and the head of the inferior nasal concha at point C (MCA2 in a positive nasal allergen provocation test). The first point is located $3.3 \mathrm{~cm}$ away from the start of the nasal cavities and the other $-4.0 \mathrm{~cm}$ away. MCA measurement as a method of assessing the most reactive areas seems inappropriate for the reason that the analysis covers an area with variable parameters. The position of MCA1 in a healthy person should be the same as wave I. If the patient suffers from severe nasal septum deviation, the area under assessment moves to the back part considerably. In contrast, MCA1 is normally located at point I before the nasal allergen provocation test and at point $C$ after the test. There are significant differences between cross-sectional areas in terms of ethnic origin, and these differences are explained by differences in the length of the nasal cavity $[5,6]$. In an attempt to determine reference values, average values were estimated for the following populations of subjects: the Caucasian race: $0.72 \pm 0.02 \mathrm{~cm}^{2}$, the Oriental race: $0.62 \pm 0.19 \mathrm{~cm}^{2}$, the African Americans: $0.88 \pm 0.22 \mathrm{~cm}^{2}$, the American Indians: $0.70 \pm 0.16 \mathrm{~cm}^{2}$, and the Asians: $0.75 \pm 0.03 \mathrm{~cm}^{2}$ [6-9].

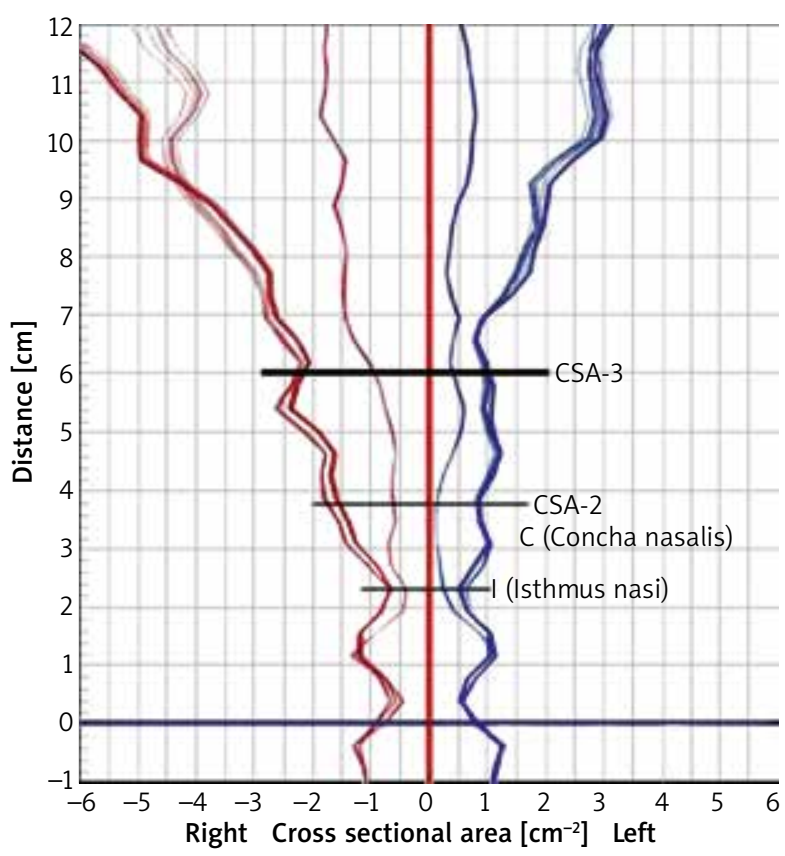

Figure 1 . Curve of relative reactivity of acoustic rhinometry

An assessment of any intranasal area may also include measuring the volume of, for example, the vestibule of the nose, the nasal cavity between the anterior and posterior edges of the nasal concha, or of the nasopharynx. Analyses by some authors measure only the vestibule of the nose. Others consider the vestibule as an integral part of the nasal cavity. Yet others exclude the first segment and measure the volume over the $2-7 \mathrm{~cm}$ segment of the nasal cavity. The volume of the nasal cavity is measured using AR most frequently for the $0-6 \mathrm{~cm}$ segment or the $0-7 \mathrm{~cm}$ segment. Volume changes can be measured more precisely if the $2-5 \mathrm{~cm}$ segment of the rhinometric curve is analysed. The reference values for the measured volume and the area under analysis are as follows: Total $\mathrm{V}=5.86 \pm 1.40 \mathrm{~cm}^{3}, \mathrm{~V} 1=1.54 \pm 0.36 \mathrm{~cm}^{3}, \mathrm{~V} 2=1.66 \pm 0.52 \mathrm{~cm}^{3}$, $\mathrm{V} 3=2.64 \pm 0.70 \mathrm{~cm}^{3}[10]$.

In contrast, a review of the literature shows that cross-sectional areas are a good alternative method of rhinometric curve assessment as it is possible to measure specific points on the $Y$ axis (located $3.3 \mathrm{~cm}$ and $4.0 \mathrm{~cm}$ from the start of the nasal cavity (CA 3.3; CA 4.0) [3, 4]. An interesting interpretation of the AR curve is proposed by Polish researchers. The start of the rhinometric curve was considered to be the first narrowing, which is wave O. Another analysed area of the broader space of the nasal cavity is, according to the researchers, positioned between waves $\mathrm{O}$ and $\mathrm{F}$ (CA is greater than $5 \%$ ) and the curve gradually becomes a narrowing (wave 1). Further calculations included only those $F$ waves which were less than 1.23 away from wave 0 . In other cases, waves $F$ and 1 were considered as no data. Interestingly, the analysis used a number of cut-off points such as: 1.15 for the 0 
wave search range only; 1.8 for the 1 and 0 wave search range; 1.23 and 2.47 as the limit values of the distance between waves 0 and $F$ and waves 0 and 1 . The CSA-3 $\mathrm{cm}$ value was measured only if waive 1 was detected. The first wave (CSA-0) visible on the curve was usually the same distance away from the start of the adapter. As indicated by the results of rhinometric measurements, that wave was detected at depths ranging from $0.59 \mathrm{~cm}$ to $0.83 \mathrm{~cm}$. However, in severe nasal septum deviation cases, the CSA-0 wave may not occur [5]. In an attempt to assess the reference values for the areas under analysis, the reference values for the cross-sectional area at specified points on the rhinometric curve are estimated as follows: $\mathrm{CSA} 1=0.73 \pm 0.27 \mathrm{~cm}^{2}, \mathrm{CSA} 2=1.74 \pm 0.47 \mathrm{~cm}^{2}$, CSA3 $=3.23 \pm 0.83 \mathrm{~cm}^{2}[10]$.

Critical factors in the assessment of intranasal areas include sex and variable anthropometric parameters of the head, which reflect the age of the subjects [10-12]. Wojas et al. have proved in their studies that the height of subjects correlated with their age: for subjects aged 6 to 18 years, the correlation coefficients were 0.89 for female subjects and 0.92 for males, which translates into the reference values for the measured cross-sectional areas. From a practical perspective, it means that regard may be had to the age of subjects when determining reference values for rhinometric measurements. The measurable rhinometric parameters correlated with the age of subjects ( $p<0.000001)$, but their correlation with sex was considerably weaker. In the case of subjects aged 12 or younger, no statistically significant differences were found between the males and the females. In terms of the surface area of the nasal valve and the average crosssectional area at the depth of $3 \mathrm{~cm}$ outside the nasal valve, significant differences between male and female subjects were identified only for subjects aged 17 years or older [5].

\section{The rhinometric curve: interpretation of the curve in nasal allergen provocation tests}

The measured (CA) points on the rhinometric curve that correspond to the cross-sectional areas of the nasal cavity at specific depths of the cavity are particularly useful in nasal allergen provocation tests (NAPTs) [13, 14]. In its consensus statement (the EAACl's Position paper on the standardization of nasal allergen challenge) [3], recognised the cognitive benefits and value of AR in the assessment of the response of the mucous membrane of the nasal cavity to an allergising agent. Moreover, the specificity and sensitivity of the technique under discussion make it a highly recognised instrument among techniques for objective assessment of intranasal areas. NAPTs with substantially positive results are assessed if the value for cross-sectional areas of the nasal cavity (CSA-2) falls by $40 \%$ or more. In contrast, NAPTs with moderately positive results are assessed if the CSA-2 value falls by $20 \%$. It needs to be not- ed that a subjective assessment of symptoms occurring during the early and late phases of the allergic reaction ( $55 \mathrm{~mm} \geq$ on the VAS scale) is critical to a complete assessment of the response of the mucous membrane of the nasal cavity. Lebel score, Linder score, Total nasal score: height $\geq 5$ points) [3]. In contrast, Amber et al. have proposed to assess the NAPT on the basis of volume changes and the minimal cross-sectional area (MCA), where a NAPT is regarded as positive if the volume/MCA value falls by $\geq 25 \%$ [10]. It seems that an assessment of the MCA value as a whole rather than MCA-1 MCA-2 separately carries a risk of interpretation in general terms: the MCA value before the NAPT is positioned in the region of the nasal isthmus and moves deeper into the nasal cavity during the early phase, and it is measured at the head of the inferior nasal concha. It seems, therefore, that an assessment after the NAPT in relation to the initial point (MCA-1), where no significant nasal patency changes occur (during the NAPT), is not really correct. Moreover, the co-existing nasal cycle may significantly affect the overall MCA value. Consequently, it seems necessary in an NAPT assessment to measure the value for MCA-2 located at the target location of the allergic reaction throughout the test process, i.e. during the initial examination, after the administration of a control solution and after local application of an allergen.

An interesting solution is proposed by Samoliński et al., who introduced the concept of relative reactivity for each point on the AR curve, i.e. for the points spaced at an interval of $3.3 \mathrm{~mm}$ determining cross-sectional areas. These points are connected to produce a reactivity curve. This curve is a representation, in percentage terms, of changes in the CA value at different points on the AR curve when the measurements for two states are compared, such as the value measured after the NAPT in relation to the initial, pre-NAPT value for the subject's nasal cavity. The reactivity of the mucous membrane of the nasal cavity is regarded as that for which an NAPT results in substantial changes to the volume or cross-sectional areas of the nasal cavity occur as compared to the initial examination or after the administration of a control solution. This reactivity is calculated according to the following formula: $\mathrm{Re}=\mathrm{CAll}-\mathrm{CAI} / \mathrm{CAl} \times 100 \%$, where Re is the reactivity value, $C A I$ is the cross-sectional area of the nasal cavity as measured during the initial examination or after the administration of a control solution, and CAll is the cross-sectional area of the nasal cavity after the NAPT. The term more reactive side is also introduced, meaning the side where an NAPT results in substantial changes to the volume or cross-sections areas of one of the sides of the nasal cavity (as compared to the initial examination or after the administration of a control solution). In order to determine the more reactive side, it is necessary to compare differences in the reactivity values between the left side and the right side and, subsequently, to decide which of the two sides is more reactive: it is the one for which the value is more negative, indicat- 
ing a stronger reaction of the mucous membrane to an allergen [4]. Determining the more reactive side of the nasal cavity is particularly important if the risk that the nasal cycle will affect the final result of the NAPT is to be minimised $[15,16]$.

It is critical for the purposes of interpreting the results of NAPTs to estimate the position of the strongest allergic reaction. It needs to be remembered that the points identified on the rhinometric curve vary depending on the age and sex of subjects, reflecting the length of the nasal cavity. The hypothesis may be proposed that the length of the nasal cavity affects the position of the area where the mucous membrane of the nasal cavity is the most reactive. In the case of subjects with long nasal cavities, as opposed to those with short cavities, the area is located at $1 \mathrm{~cm}$ to the back end of the AR curve. It is usually the case that no significant changes to point I are identified in terms of the anatomical or functional structure of the assessed area (the nasal isthmus, the osteochondral framework) [17-19]. Another aspect is the determination of a corresponding segment with the highest relative reactivity. Studies by Samoliński show that the segment is positioned $3-4 \mathrm{~cm}$ away from the end of the adapter, known as the ascending arm of wave C. The more intensive changes in the cross-sectional areas of nasal cavities are found over a $3-\mathrm{cm}$ segment after point C (CA-C3). It is easy to find this parameter in a figure showing the results of AR measurements. This new method of assessing the results of NAPTs has been shown to be consistent with the clinical condition of the patient and the patient's self-assessment during the test.

\section{Conclusions}

$A R$, being an objective nasal patency measurement technique, is an excellent alternative in the diagnosis of nasal cavity diseases. Assessed with the use of crosssectional areas of the nasal cavity over appropriate segments of the rhinometric curve, it seems to be a faithful representation of changes in the course of the NAPT.

\section{Conflict of interest}

The authors declare no conflict of interest.

\section{References}

1. International Consensus Report on Diagnosis and Management of Rhinitis. International Rhinitis Management Working Group. Allergy 1994; 49 (19 Suppl): 1-34.

2. Krzych-Fałta E, Furmańczyk K, Samoliński B. Specificity and sensitivity assessment of selected nasal provocation testing techniques. Adv Dermatol Allergol 2016; 33: 464-8.

3. Augé J, Vent J, Agache I, et al. EAACI Position paper on the standardization of nasal allergen challenges. Allergy 2018; 73: 1597-608.
4. Samoliński B. Analiza wyników rynometrii akustycznej na potrzeby diagnostyki rynoalergologicznej. Habilitation thesis. Wydawnictwo Naukowe Scholar, Warszawa 1998.

5. Wojas O, Szczęsnowicz-Dąbrowska P, Krzych-Fałta K, et al. Changes in the cross-sections of the nasal cavity assessed by acoustic rhinometry in the study population as a guideline for attempts to standardize nasal provocation tests. Adv Dermatol Allergol doi.org/10.5114/ada.2021.105361.

6. Garcia GJM, Hariri BM, Patel RG, et al. The relationship between nasal resistance to airflow and the airspace minimal cross-sectional area. J Biomech 2016; 49: 1670-8.

7. Morgan NJ, MacGregor FB, Birchall MA, et al. Racial differences in nasal fossa dimensions determined by acoustic rhinometry. Rhinology 1995; 33: 224-8.

8. Gurr P, Diver J, Morgan N, et al. Acoustic rhinometry of the Indian and Anglo-Saxon nose. Rhinology 1996; 34: 156-9.

9. Huang ZL, Wang DY, Zhang PC, et al. Evaluation of nasal cavity by acoustic rhinometry in Chinese, Malay and Indian ethnic groups. Acta Otolaryngol 2001; 121: 844-8.

10. Trindade IE, Gomes Ade O, Sampaio-Teixeira AC, et al. Adult nasal volumes assessed by acoustic rhinometry. Braz I Otorhinolaryngol 2007; 73: 32-9.

11. Distinguin L, Louis B, Baujat G, et al. Evaluation of nasal obstruction in children by acoustic rhinometry: a prospective study. Int J Pediatr Otorhinolaryngol 2019; 127: 109665.

12. Cunha R, Nazareth A, Rino-Neto J, et al. Acoustic rhinometric evaluation of the nasal cavity after rapid maxillary expansion. South Eur J Orthod Dentofac Res 2018; 5: 6-11.

13. Laine-Alava MT, Murtolahti S, Crouse, UK, et al. guideline values for minimum nasal cross-sectional area in children. Craniofac J 2018; 55: 1043-50.

14. Ibon EG, Almudena TM, Salas M, et al. Comparison of diagnostic accuracy of acoustic rhinometry and symptoms score for nasal allergen challenge monitoring. Allergy 2021;76: 371-5.

15. Pantin CT, Southworth T, Wetzel K, et al. Reproducibility of nasal allergen challenge responses in adults with allergic rhinitis. Clin Pharmacol 2019; 11: 67-76.

16. Pepper AN, Ledford DK. Nasal and ocular challenges. J Allergy Clin Immunol 2018; 141: 1570-7.

17. Gotlib T, Samoliński B, Grzanka A, Balcerzak J. Dynamics of early phase congestion after nasal allergen provocation. Rhinology 2008; 46: 52-5.

18. Gotlib T, Samoliński B, Grzanka A. Bilateral nasal allergen provocation monitored with acoustic rhinometry. Assessment of both nasal passages and the side reacting with greater congestion: relation to the nasal cycle. Clin Exp Allergy 2005; 35: 313-8.

19. Samoliński B, Rapiejko P, Krzych-Fałta E, et al. Standardy wykonywania donosowych prób prowokacyjnych. Adv Dermatol Allergol 2010; 273: 141-60. 